\title{
RESEARCH ON ONLINE PUBLIC PARTICIPATION AND ONLINE PLATFORM CONSTRUCTION FOR HISTORICAL BLOCK PROTECTION IN THE DATA AGE
}

\author{
Yang YANG, ouw@deakin.edu.au; Australia \\ Jilong ZHAO, zlll0w@sdjzu.edu.cn; China \\ Qian SUN, 573295334@qq.com; China
}

\begin{abstract}
With the implementation of benefit-oriented urban renewal, many historical blocks have lost their identifiability. The historical block is an external entity that is a presentation of urban culture. The disappearance and homogenization of the historical block have directly led to the city losing its place attachment, with the overall identifiability of urban areas becoming weakened. Therefore, it is essential to develop a strategy to protect historical block identifiability in urban planning. Traditional urban planning is dominated by government and developers and is highly dependent on planners' experience-based judgment but lacks quantitative analysis of public participation. As a result, it is difficult to carry out an objective and comprehensive analysis when facing the complicated situation of historical blocks. The issue of public participation has become an essential issue in the process of urban construction and renewal in China and other developing countries. Based on an analysis of the concept and the characteristics and techniques prevalent in the data age, this article discusses (1) the method and mechanism of public participation in the protection of urban historical blocks and (2) the content and structures of public participation platforms for historical districts. Research indicates that the application of cloud technology and reasonable platform design is the focus of public participation in historical block protection, which can change public participation from passive to active, from "lagged" to "synchronized".
\end{abstract}

\section{Keywords}

public participation, online platform, historical block protection, data age

\section{Introduction}

In many developing countries, there is an urgent need for an applicable public participation platform for the renewal and protection of urban historical blocks (Wei et al.,2015; Cheng et al., 2015). On the one hand, this need is born from the gradual disappearance and total commercialization of blocks that carry historical value and memory. On the other hand, under the original "top-down" model of historical block protection and development, the 
public can only be passive and accept renewal policy that has already been decided. Among citizens, the government, and developers, it is easy for issues like housing demolition and relocation to occur (Liu, 2007). Therefore, for the renewal and protection of urban historical blocks, we need to not only preserve the original historical memory but also develop an open and effective public participation platform.

However, there are many severe deficiencies regarding the modes and technologies of current historical block protection (Feng and Li, 2013). The renewal and protection of urban historical blocks is a complicated urban social issue and requires communication channels and time to organise and update social relations. On the one hand, existing urban public announcement and online comment platforms are often vacant, making public participation an institutional decoration to a large extent. On the other hand, the results obtained from traditional public participation modes are mainly based on voting and writing, which are difficult to analyse and directly categorise (Liu, 2007).

New media platforms in the data age stimulate public awareness of independent participation. In the age of digitalisation and interconnection, with the popularity of new media such as microblogs and WeChat, Internet public participation events such as the PX project boycott event have had an impact on government decision-making, and China has begun to see the burgeoning of citizen network participation politics (Dai et al. 2014). PX is the abbreviation of xylene, and the PX project involves the production of xylene. In recent years, some people have worried that PX is harmful to human health and have organised walks and parades through the Internet and other means to boycott a PX project planned for construction in a locality. Since 2007, such incidents have occurred successively in Xiamen, Chengdu, Dalian, Ningbo, Kunming, and other places. The incidents had a certain impact on the government's decision-making. Most of the above cities announced the termination of relevant PX projects. Meanwhile, the protection of historical blocks has received extensive attention. Besides, with smart handheld devices becoming the main terminals of people's lives, daily life and social affairs are demonstrating diversity and immediacy, gradually changing the mode of public participation in the protection of urban historical and cultural resources from passive to active (Zhou et al., 2012).

Therefore, in view of the current situation of urban historical block protection in China, the establishment of a public participation platform based on new media can enhance the participation of all parties, increase the diversity of public participation modes, and improve cultural information about urban historical blocks available from various channels. This study explores the three aspects of public participation theory, mechanisms, and a platform of the historical block protection, with empirical research conducted in combination with an examination of existing cases.

\section{Characteristics of Public Participation in Historical Block Protection in the Data Age}

\subsection{Interpretation of basic concepts}

A historical block refers to a block with a certain scale of historical relics and complete historical features. It is the material carrier of social life and cultural habits during a specific 


\section{Online Public Participation \\ Platform Construction for Historical Block Protection}

period. The "Athens Charter", the "Venice Charter" and the "Special Measures for the Protection of Ancient Capitals' Historical Features" all proposed that an essential aspect of historical block protection is the overall protection of the historical, cultural, material, and living environment of the blocks. In the protection of historical blocks, attention should be paid to the structure and customs of subjects and their diverse use of space.

Arnstein (1969) believed that "public participation is a right of the public". In his opinion, public participation can be divided into eight steps: manipulation, guidance, notification, consultation, persuasion, cooperation, authorization, and public control. Public participation in the historical block protection is essentially a multi-step redistribution of the protection rights of diverse subjects such as residents, social groups, non-profit organizations, and individuals (Lane, 2005; Choguill, 1996).

With the increasing popularity of interactive network platforms and smart mobile terminals, more and more commercial and public platforms have begun to feature personalized content based on users' collected preferences and requirements. The real-time interaction of a large number of diverse subjects with information using new mobile media as the primary tool is the main feature of the data age (Yu and Mao, 2015).

From the above analysis, it can be seen that the interaction and integration of multi-subject activities and information, as well as the redistribution of multi-subject protection rights, are the integration basis of historical block protection, public participation, and new media.

\subsection{Public participation in historical block protection based on "Cloud"}

The Internet provides an interactive interface for public participation in historical block protection. Feng and Li analysed the impact of public participation on the restoration and renovation of Beijing's "Zhonggulou" Square (Feng and Li, 2013). Through the network media platform, the renovation of the "Zhonggulou" Area received widespread attention, and some government decisions were adjusted and modified in response (Feng and $\mathrm{Li}$, 2013). In "Internet-based planning information exchange platform and public participation platform construction", Zhou (2012) stated that the network-based public participation platform is characterised by an "Internet-based user interface" and "information interaction with users". On the one hand, the system transmits visible and understandable results and information to the public; on the other hand, it gathers public feedback and quickly processes it.

New media is an effective tool to intervene in the protection mechanisms of public historical blocks. In "The Mechanism and Exploration of New Media Involvement in Public Participation for Historical Blocks Protection", Wei et al. (2015) introduced attempts at public participation in historical block protection and renewal projects using new media technologies. From the earliest WebGIS to WeChat public accounts and the Heritage Protection APP, he proposed that new media has changed the way information is disseminated and created opportunities for public participation. Through new media, platforms for display and exchange can be built to promote public participation, lower the threshold of public participation, and enhance enthusiasm for public participation.

The application of cloud technology has opened up the era of "cloud" distribution for public participation in the protection of historical blocks. For example, the Beijing Planning and Design Institute developed the CitylF Urban Planning Cloud Platform and proposed that due 


\section{Online Public Participation \\ Platform Construction for Historical Block Protection}

to its social characteristics, the new era is a "micro-era". The CitylF Urban Planning Cloud platform was sponsored by Beijing Research Institute of Planning and Design (Chu et al. 2015). It provides different data and information services for various groups such as citizens, the government, and planners, with the aim of bringing together urban data, planners' wisdom, citizens' bottom-up power and government's top-down power to form a planning cloud platform and a platform for discussing the urban future. To acquire and integrate data, a "cloud platform" that can realize interactive sharing, multi-disciplinary collaboration, and data aggregation should be built. Such a platform consists of three layers of resource platforms: one is an analysis platform that analyses and processes big data; the other is a planning platform that collects the professional intelligence of planners; the third is a dynamic platform for community governance from the bottom up. On this basis, the platform provides three different operational interfaces for three groups: the government, planners, and citizens (Chu et al. 2015). Based on the "cloud platform", the interaction and integration of multi-agent activities and information and the "cloud" distribution of protection rights can be fully realized for the protection of historical blocks.

In short, the Internet and new media are effective interfaces and tools for the "cloud" distribution of public participation rights. The cloud platform has opened up a vast network space for public participation in historical block protection.

\section{Online Technology and Platform Construction for Public Participation}

\subsection{Limited participation "offline" and unlimited participation "online"}

The traditional historical block protection mode can be divided into four categories according to the dominant party. In the first category, the government is dominant. The primary role of the public is to be informed and influence the government's decision-making, such as with the reconstruction of Warsaw after World War II or the renovation and restoration of Beijing's "Juer Hutong" (Hu et al., 2014). In the second category, civil organizations and individuals are dominant. This means that civil organizations or individuals guide the public to participate in the protection of historical blocks, such as the British "Ancient Building Protection Association" or the "Enning Road Academic Concern Group" during the renovation of Enning Road in Guangzhou (Wu, 2014). In the third category, the public spontaneously and actively participates in the protection of a district, such as in the "Community Vision Planning" in Halifax, Canada or the construction of the historical community of Dadaocheng in Taipei City (Macaulay et al., 1999; Xu and Liu, 2012; Liang, 2014). In the fourth category, multiple parties collaborate. This collaboration is initiated and implemented by the above parties, and the public is widely involved and becomes essential. A typical case is the Hong Kong Central Market Conservation Plan.

Among the four methods discussed above, public involvement in the protection of historical blocks can be classified into three main categories: First, property owners, who have a direct interest in the daily management and renovation of historical blocks and are mainly local residents, often do not need guidance and inducement. The space in which they live is the material element of the historical block, and the customs and community structure are also important aspects of protection (Hu et al., 2014). The second is the common interest in the 
block, which mainly refers to experts, scholars, professional organizations, and nongovernmental organizations relevant to citizens' public interest in the block. These entities mainly focus on the value of cultural research and the development, utilization, and valuation of historical blocks. The participation of such entities can effectively avoid the intensification of social conflicts or the excessive commercialization of neighbourhoods. The third category includes other individuals without a direct interest. Since there is no direct interest, they generally do not pay attention to historical acts and do not act spontaneously. However, with the development of society, the role of public opinion and action is becoming increasingly important. Through proper guidance, this will benefit the protection and development of historical blocks (Xu and Liu, 2012).

The public mainly participates in the protection of historical blocks in the following three aspects. First of all, it participates in the collection and protection of historical information, the investigation of historical resources, and the completion of data, all of which are essential work in the process of historical block protection. In addition to historical surveys and physical surveys, genealogical information and photos of local residents can provide information about the neighbourhood. This complements the physical form of the building and contributes to complete historical information about a block. Secondly, the public participates in the positioning, design, and implementation of historical block protection planning before the formulation of the plan, so residents and other members of the public have a detailed understanding. Based on the plan, suggestions are put forward. In the design process for a protection and renovation plan for a historical block, the public acts as a judge. After they understand the design plan, people make comments and suggestions, or vote on whether a certain transaction should be implemented (Zhou et al., 2012). Third, the public also participates in the cultural and tourism development of historical blocks. Local residents can preserve the social structure and customs of a neighbourhood and participate in the development of cultural and creative industries and tourism. On this basis, civil organizations and individuals can also hold culture exhibitions and other events promote local culture. In this way, on the one hand, the local culture is protected, and on the other hand, the social economy is developed. In this way, the benign development, protection, and transformation of historical blocks are realized.

At present, there are four main ways for the public to participate in or influence the protection of historical blocks. The first is information browsing in which relevant government departments and other entities publicize information and promote exhibitions to the public, but there is no interaction and poor participation (Liu, 2007). The second is the collection of opinions in which relevant departments obtain questions and opinions through on-site investigations and questionnaires and make plans based on this information, and the public participates in the protection of historical blocks (Zhou et al.,2012; Brown and Raymond, 2014). The third is collaborative participation in which the public participates in planning and design through meetings and expert workshops organized by the government, institutions, or non-governmental organizations in an effort to encourage the participation of residents, civil organizations, the government, and other subjects. This model involves government guidance and multi-subject participation and is the main direction of public participation in the protection of historical blocks in China. The fourth is public leadership in which self-governing community organizations and advisory committees collect public concerns and develop a community vision and action plans on behalf of community 


\section{Online Public Participation \\ Platform Construction for Historical Block Protection}

residents and the general public to enable them to participate in planning, construction, and other relevant departments through negotiation and communication (Liu, 2007).

However, in combination with China's actual conditions and existing practices, the following problems must be solved to promote public participation in the protection of those as mentioned above historical in China. First, awareness of public participation is weak, and the Chinese public has little relevant experience with participation (Liu, 2007). Second, the main form of public participation is unclear. For a long time, Hong Kong, Macao, Taiwan, Europe, and the United States had relatively complete public participation systems that included the government, non-governmental organizations, residents, experts, and professional institutions. The public participation system in China remains unclear, and the power of participating entities is not clearly defined or distributed. Third, the content of public participation is limited. At present, management and renovation of most of the historical blocks in China are mainly led by the government, and the designers and developers are entrusted with carrying out planning and transformation. The public plays only the role of being informed, and there are no laws and regulations. Usually, a program has been basically decided when it is publicized, so public participation is of little effect. Fourth, there is a single method of public participation. At present, there are only methods such as on-site interviews, questionnaires, symposiums, and public announcements, and most of the public is passively involved, with one-way notifications. Fifth, there is no feedback mechanism. The key to public participation is interaction. At present, many historical block protection plans do not allow for feedback, so naturally, the public cannot influence decision-making or the formulation of plans and policies.

\subsection{Online public participation platforms in the data age}

New technologies emerging in the data age provide new methods to solve the above problems. Under the support of new technologies, management and operation models such as "crowdfunding", "PPP", "Joint planning and Joint management" began to be introduced into historical block protection (Yu, 2014). The online public participation platform not only provides citizens with convenient access to participate in the decision-making process but also provides opportunities for planners and citizens to interact (Yu, 2015). Compared with traditional public participation, online public participation based on data technology has the following advantages:

Historical information dissemination methods are diversified. The development of new media and the popularity of mobile smart devices have enabled the rapid dissemination of information, and new media directly push content to personal devices that spread quickly among the public through forwarding. Using QR codes, the public can link to WeChat, Microblogs, news pages, and other platforms to obtain and forward information about historical block protection anytime, anywhere.

Diversified ways of participation. In traditional public participation, feedback is mainly based on questionnaires and voting, and data is in a single form. In the era of data-based information, mobile phones, tablets, and desktop computers can be used to create online maps of historical block maps by means of webpages, WeChat, Microblogs, and other platforms. The public can directly participate in the design, make comments, or take photos of historical blocks with location information that reflect the status quo. Through data 


\section{Online Public Participation \\ Platform Construction for Historical Block Protection}

information platforms, the government can also timely obtain public opinions on the protection of historical blocks and realize interactive information.

Participation of diverse groups. From the original government, residents, and experts to the whole society, participants include students, teachers, folk history protectors, nongovernmental organizations, and other individuals and groups concerned with the protection and development of historical blocks. This reflects the popularity and whole process of public participation.

Diversified content of information. A significant feature of new media is the diversity of content and form. Data platforms can use any digital media content such as videos, 3D models, and panoramic images. New media and the SoLoMo service have expanded the way information is delivered and allows it to be presented more intuitively, allowing the public to interact with content. SoLoMo is composed of the first two letters of three words: "Social", "Local", and "Mobile". "So" stands for all kinds of social networks, such as Sina microblogs and Facebook, "Lo" stands for all types of services based on geographical location, and "Mo" stands for mobile Internet. SoLoMo is the combination of the three.

Diversified data resources. The era of big data has promoted governments' database construction and data availability, and many government agencies are digitizing information and gradually making it public. Data-based information platforms can use the cloud to access various types of information. For example, in the historical block protection process, the land management database of the national land department, the project management and design plan library of the planning department, and the database of historical building protection information can be linked. A variety of content can be added to online maps to increase the public's understanding of issues.

\subsubsection{Technical means of public participation in the data age}

Specifically, in the data age, there are many technical means that can be applied to public participation in historical block protection.

The first is the QR code. QR code transforms textual information such as serial numbers, website addresses, and item characteristics into QR black and white images that can be directly recognised by computers and can be read by simple image input devices (such as mobile phones with a camera function). With the help of the QR code, the functions of information acquisition, web page jumping, and mobile payment can be realised. With the popularity of smart devices such as mobile phones, QR codes have become an essential way for the public to obtain information. In recent years, Shanghai, Harbin, and other cities have begun to post QR code on the nameplates of historical buildings, conveniently allowing the public to connect to a detailed network introduction page and solving the problem of limited nameplate content. Moreover, the public can directly comment on, suggest, map, or upload other relevant information, such as old photos and documents, through QR codes. In this way, the public can participate in the collection of information on historical blocks.

The second is the web service API. Network Service API is an open platform for network services. The allocation of traditional Internet resources is unbalanced, and a large amount of data generated by users is gathered by enterprises or institutions, resulting in other enterprises or industries being limited by the lack of user resources and data. To realize the sharing of resources, many enterprises have set up an open platform for network services to 
open up their own resources to ordinary developers to a limited extent. The web service API is a resource interface provided by data holders (such as large Internet companies) that enables developers to access or use their resources. With the opening of the web service API, government agencies and public organizations will be able to create online public participation platforms more easily. For example, through the Baidu map API, developers can use Baidu map data and apply them to various map data services. The system that the author of the present paper developed offers costs savings in terms of data storage and transmission, reduces the time required to write a large amount of program code, and also transforms the main work of the public participation platform builder from collecting data and writing code to conceptualizing and realizing the function logic of the public participation platform. The public can also use the API service to browse the city's historical information and map markers and engage in public discussion and other participation behaviours. This reduces the public's learning costs and promotes public participation.

The third is virtual display technology. Virtual reality (VR) is a technology that allows users to immerse themselves in a computer-generated, interactive three-dimensional environment. Augmented reality (AR) is a technique for calculating the position and angle of camera images in real-time and adding corresponding images to combine real images with virtual images. In the historical block protection process, if the general public, which lacks professional knowledge and experience, is involved, there must be an intuitive and easy-tounderstand way of displaying information. Virtual displays based on computer graphics technology not only allow the public to intuitively understand history and culture but also display historical block renovation plans, enabling the public to understand design plans and make suggestions. In recent years, data acquisition technology has advanced by leaps and bounds, with advancements including three-dimensional laser scanning and panoramic photography. With the development of network technology, the threshold of $3 \mathrm{D}$ data display is becoming increasingly low and low, particularly through WeChat, Microblogs, APP, and other platforms that allow viewing and access anytime, anywhere. In recent years, the VR and AR6 technologies, which have received much attention from the industry, have allowed the public to understand historical streets and their renovation plans immersively and to provide opinions and suggestions.

The fourth is big data technology. Technologies such as data mining and cloud computing are rapidly evolving. People are increasingly using mobile smart devices, generating a lot of spatial data. Spatial distribution and semantic analysis of these data can be used to derive behavioural patterns and the views of the public. On the other hand, public participation will generate necessitates downloading and uploading massive data, which requires excellent data processing algorithms. At the same time, cloud platform technology is rapidly evolving, improving data processing efficiency and ease of use by linking and integrating different databases. A typical example of big data technology is Location Based Social Network (LBSN). LBSN is a social network based on location. Compared with traditional social networks, LBSN can track and share real-time location information of people in addition to using network technology to realize virtual contact between them. LBSN uses the massive data it collects to combine data mining and cloud computing technologies and analyse the public's use of historical buildings or neighbourhoods in a certain city so that the public "unconsciously" participates in history. The protection and renovation of blocks through this method has a positive impact. 


\subsubsection{Public participation platform carriers in the data age}

At present, many network service platforms have integrated the above-mentioned key technologies. Online platforms for public participation in historical block protection can be completely built on the basis of "the platform of platforms" using "carrier platforms" to avoid a large amount of time and energy required for bottom-level development. At present, there are the following relative mature "carrier platforms" in China:

One is the information visualization web page. With the development of technology, the information content of web pages has moved from graphics and videos to interactive and operable web pages. The information conveyed by web pages should be easy to understand as well as visual and interactive. This idea also corresponds to online public participation platforms in historical block protection.

The second is the microblogs. Its main advantage lies in its broad range of audiences. Through Microblogs, the public can understand the protection and renovation plans for historical blocks. Recent years have witnessed the phenomenon of the destruction of outstanding historical buildings in many cities. Local residents, experts, and scholars have appealed to society through Microblogs, forming a public opinion environment that enables the government to listen to the opinions of people and accordingly adjust their policies and programs.

The third is WeChat, mainly WeChat public numbers. The WeChat public number service is open to organizations, groups, and individuals. Based on this platform, specific functions can be developed to realize information push and interactive feedback. According to a 2015 report by Tencent, WeChat covers more than $90 \%$ of smartphones, and monthly active users reached 549 million. Its popularity is evident. Using a WeChat public account, it is possible to build a historical block protection participation platform that can be used whenever and wherever possible. Such a platform makes the public willing to participate more actively in discussions on urban construction affairs and policies. Planning departments can also collect and understand public opinions and adjust their historical district protection policies and plans.

The fourth is the independent APP (Application). As APP relies on mobile devices such as mobile phones, it has greater flexibility than traditional web browsing or entity modes. Compared with microblogs and WeChat public number, APP is relatively independent and unrestrained and can realize more abundant and easy-to-use functions. For example, using the Tianjin historical style building APP, the public can combine the GPS function of mobile phones to locate historical buildings conveniently. At the same time, explanations of the historical background of a building or block help to cultivate the public's sense of historical protection. At the same time, the discussion platform can be set up through the APP, and the public can send feedback information to the planning bureau and other departments or institutions through the APP.

These platforms have significant differences in information collection mode, information storage mode, and interaction mode. Therefore, different platforms have different advantages and disadvantage in terms of participatory degree, content richness, and types of participation modes (see Fig 1). Thus, the design of online public participation platforms 
for historical blocks protection needs to design an independent technical framework based on specific content.

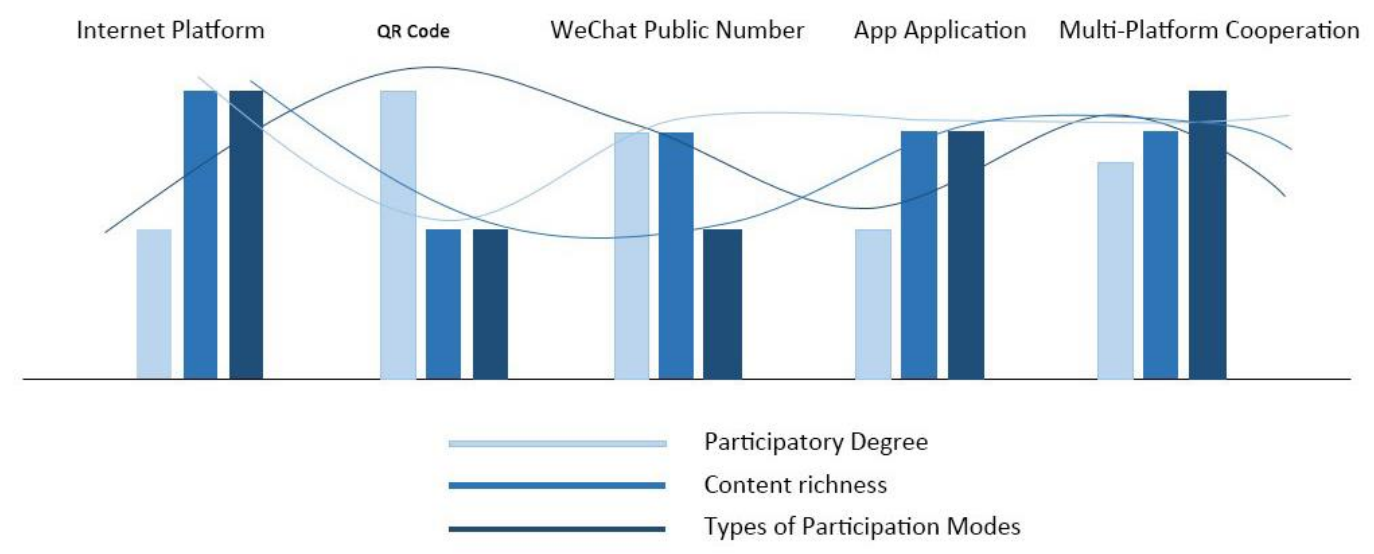

Fig. 1 Comparison of Public Participation Platform in the Data Age (Source: Author)

\subsection{Construction of online public participation platform for historical block protection in the data age}

\subsubsection{Content of public participation platforms for historical block protection}

There are three main types of users involved in online public participation platforms for the protection of historical blocks. The first is the government, developers, and designers that build, manage, and operate the platform. They are also important users of the platform and have the responsibility of communicating to the public the orientation, objectives, background, and presentation of design schemes for the protection or renovation of historical blocks. On this basis, public opinions are collected, and feedback is given. The second is community representatives, who are direct participants. This includes representatives of residents and industry, who use the public participation platform mainly to gather public opinions and questions and transmit them to relevant departments. The third is indirect participants, which includes civil protection forces, experts and scholars, academic institutions, civil scholars, cultural security personnel, and non-governmental organizations. These participants use the platform to organize diverse activities and establish links between the general public and government agencies, which are significant forces that cannot be ignored in the protection of historical blocks. In addition, the general public in urban and other areas can gain understanding, engage in discussion, and provide feedback through the public participation platform throughout the process of protection and renovation of historical blocks, thereby influencing policies and planning.

In view of the functional requirements of the types mentioned above of users of online public participation platforms, such a platform should include the following functional modules:

The first is the sub-platform of historical information collection and display. This subplatform has four functions: The first is collecting historical and cultural information and 
relevant historical pictures, text, audio, and video from members of the society and establishing a historical information resource bank concerning the blocks. The second is publicizing historical and cultural displays using virtual display technology to show the history of the blocks to the public. The third is the recommendation and identification of historical buildings. Recommendations, voting, and other means are recommended activities to ensure excellent historical buildings or blocks, and professionals are invited to investigate and identify the buildings. The fourth is the social supervision of historical sites, which involves reporting destructive behaviour in an effort to protect cultural relic and sites and ensure well-preserved historical buildings by uploading photographs and commenting on them.

The second is the sub-platform of planning design and implementation management, which includes four functions: The first is the preliminary investigation and discussion of planning. The public's questions and opinions are collected through WeChat, microblogs, and other means and are used as the basis for the formulation of the planning scheme. The second is the design and discussion of the planning scheme. Through this platform, the public can understand in detail the current situation and future orientation of the blocks. At the same time, the public can put forward opinions on planning through stippling, discussion and other means. After collecting opinions of the public, they can be analysed. This forms the basis of the planning scheme design. The third is the evaluation of planning efforts. Through the public participation platform, the public can participate in evaluating the results through voting, commenting, and other means and comprehend feedback from public opinion. The fourth is the implementation and management of planning. The government formulates short-term implementation plans, initiates activities and discussions with the public through the public participation platform, and coordinates conflicts between construction and residents' lives. At the same time, the construction situation is announced to the public in a timely manner. This allows "cooperative co-construction" between residents and the government to be gradually realized.

The third is the sub-platform of cultural activities and tourism development in historical blocks, which includes three functions: The first is the interactive display of tourism information, which allows tourists to quickly and conveniently understand tourism information on blocks and realize an "experiential" tour. Second, cultural and creative industries are displayed to propagandize and promote cultural and creative industries in historical blocks. The third is organizing and publicizing cultural activities, which enriches the cultural life of the blocks by inviting scholars or artists to give lectures and seminars.

3.3.2 Technical framework for public participation platforms in the data age According to the mode, subject, and content of platform participation, the technical framework of such a platform in the data age is shown in Fig. 2. 
Public Participation Subject in Historical Block Protection

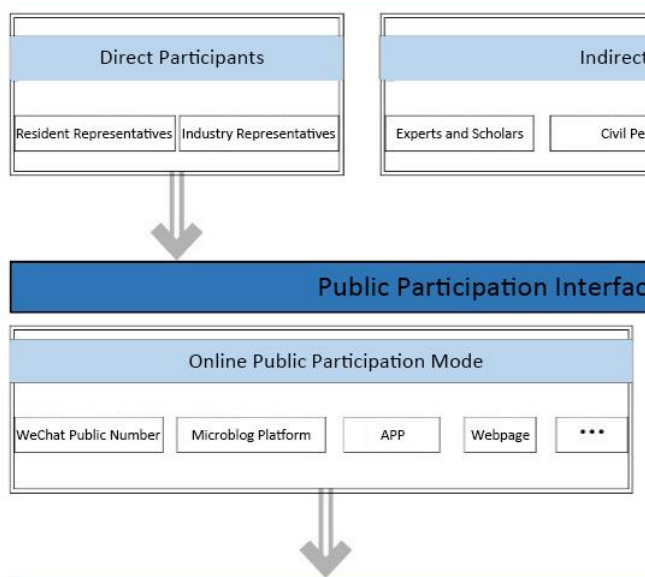

direct Participants

Composition of Public Participation Platform for Historical Block Protection
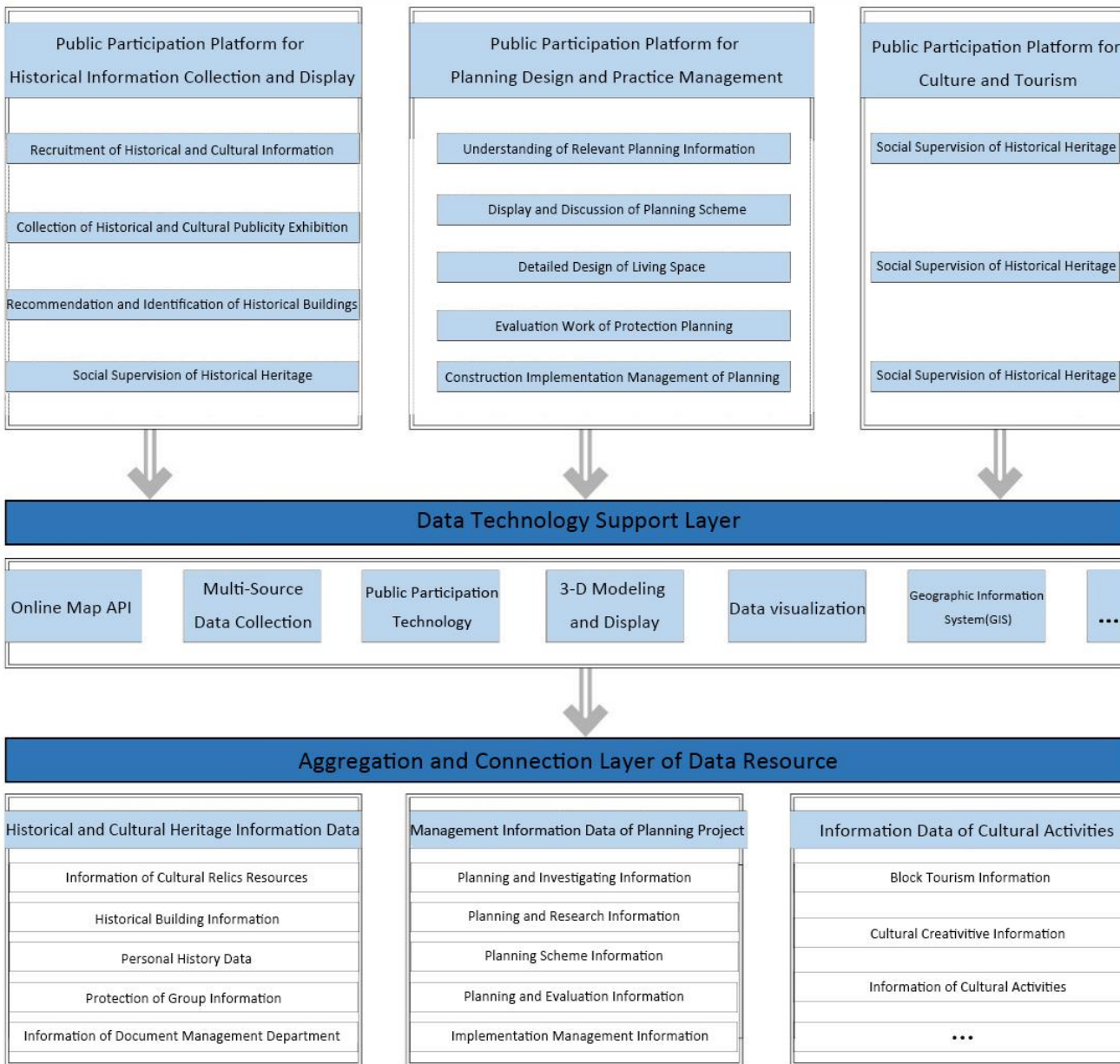

Public Participation Platform for Culture and Tourism

Social Supervision of Historical Heritage

Social Supervision of Historical Heritage

Social Supervision of Historical Heritage

Dechnology Support Layer

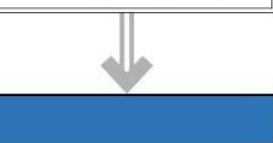

\begin{tabular}{|l|l|l|l|}
$\begin{array}{c}\text { ublic Participation } \\
\text { Technology }\end{array}$ & $\begin{array}{c}\text { 3-D Modeling } \\
\text { and Display }\end{array}$ & Data visualization & $\begin{array}{c}\text { Geographic Information } \\
\text { System(GIS) }\end{array}$ \\
\hline
\end{tabular}

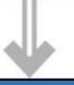

ggregation and Connection Layer of Data Resource

Fig. 2 Technical Framework of Public Participation Platform in Data Age (Source: Author)
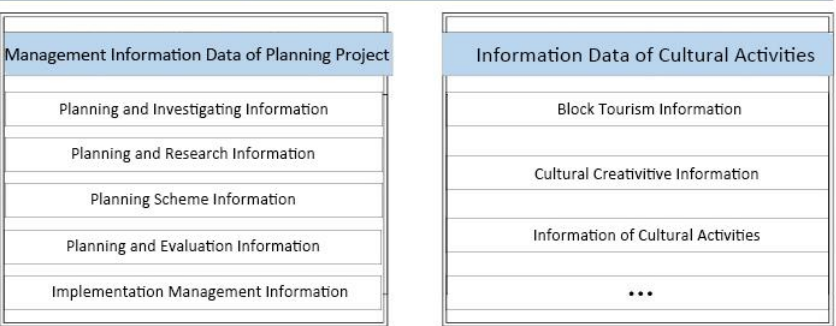


\section{Practical Analysis of Cases of Public Participation Platforms}

\subsection{Public Participation Platform for Historical Resource Management - - Beijing Historical and Cultural Heritage APP}

Beijing has a significant cultural heritage distributed in large and small streets and communities. However, geographical locations and other information are mainly stored in cultural relics in department or institution exhibits, and it is difficult for the public to have a comprehensive understanding of them. At the same time, many historical relics are located deep in the streets and alleys, and it is difficult to visit them only with the textual address information. In addition, many historical relics are scattered and cannot be fully monitored and managed. The uncivilized behaviours of individual tourists and their illegal demolition by developers often cannot be stopped in time. Besides that, threats that historic buildings face can be varied and extraordinary. Due to the prevalence of wooden building materials, corrosion and aging are severe problems of many historic buildings. The fact that ancient Chinese buildings usually have delicate architectural details makes corrosion and aging more destructive. Structural details such as tenon and mortise joints become corroded by rainfall or damaged by worms even with a protective layer of lacquer on the surface, and paints on these buildings may be damaged by the weather, including rain, snow, wind, and even sunshine. Another problem of wooden structures is the risk of fire, and the situation is especially severe in light of the traditional compact arrangement of buildings in China. Fire usually spreads rapidly both inside the building and between buildings, and the narrow streets limit the success of modern fire-fighting methods. Therefore, building a platform for the public to understand the distribution of cultural relics and related information intuitively and to provide timely reports on the damage of cultural relics has become an important direction of public participation.

The Beijing Cultural Heritage APP developed by the Technology Innovation Center of Tsinghua Tongheng Planning and Design Institute is such a platform. This platform includes information on 336 existing cultural protection sites in the eastern and western urban districts of Beijing, including the World Cultural Heritage of the Forbidden City and the Temple of Heaven. The software uses Baidu maps as a geographic information platform and displays multi-level cultural protection content through different layers.

In addition to showing the overall distribution of heritage sites, the software also displays information for each heritage site separately. Clicking on the corresponding icon allows users to view detailed information, including relevant photos. Using the API of Baidu Street View Map, the street view around a building can be seen, creating a stronger sense of presence.

In addition to providing images and textual information on cultural heritage sites, the platform also encourages the public to upload photos they have taken and add comments to enhance interactivity. The platform also includes an alarm function for cultural relics, providing a channel for public supervision, so that people can directly report the damage to cultural and stop illegal demolition and illegal construction in a timely manner. 


\subsection{Public Participation Platform for Planning, Design, and Implementation Management-- WebGIS Network Public Participation Platform for Beijing's "Zhonggulou" Area}

The "Zhonggulou" area is located near the "Di'anmen" area, which is in the eastern urban district of Beijing, on the city's central axis. Along with promotion of the renovation of old urban districts in Beijing, an upgrade plan for the "Zhonggulou" Area was proposed in 2010. According to the plan, the nearest Hutongs from "Zhonggulou" were to be demolished. The news spread rapidly through the Internet, and a large number of experts and scholars began to call for the protection of the texture of Hutongs and the landscape based on the central axis. Under this circumstance, in December of the same year, the renovation project was officially terminated, and the scope of the renovation was reduced to less than one-tenth the original, for a partial renovation.

During the renovation process, in addition to the appeals of experts and scholars and media reports, a special team played a huge role, namely the "Zhonggulou" Area team. The team intended to safeguard the rights and interests of local residents and continue the area's history and culture. Most of the team members met through microblogs, and a large number of student volunteers were recruited. They collected data through household surveys, interviews, the study of ancient books, and other means and disseminated protection information through microblogs and other platforms. After analysing the collected data, they developed a public participation platform for the "Zhonggulou" reconstruction community with the help of WebGIS technology. This allowed the team to integrate all kinds of data into the platform to facilitate residents and the public to understand the current situation and future of the area fully. The platform also enabled discussion and voting to investigate and analyse public opinion.

The participation platform relied on research and data collation and used WebGIS as a technical means to present the results in the form of an interactive platform for community residents and the public. The platform was spontaneously formed by non-governmental social teams. Although it did not play a decisive role in the development of the area, it provided an interactive channel for social cooperation and community participation to facilitate residents to understand policy information and preserve the style of the blocks through a visual platform.

\subsection{Public Participation Platform for Cultural Activities and Tourism Development - -WeChat Public Number for "Slow Products in Shanghai Hunan Community" on Wukang Road, Shanghai}

Wukang Road, Hunan Street, is located in the centre of Shanghai. Due to its rich historical and cultural anecdotes and buildings, it is known as the "Celebrity Road that condenses the history of Shanghai in the past 100 years". There are 14 excellent historical buildings along the road and 37 preserved historical buildings. On June 11, 2011, it was selected as a "Famous Street of Chinese History and Culture".

On June 15, 2014, Shanghai piloted the "E-age" historical building protection project in the Hunan community, which involved posting a $Q R$ code on the nameplate of excellent historical buildings and creating the "Slow Products in Shanghai Hunan Community" WeChat 
platform. That was the first in China, and some other cities in China have also promoted it since then.

Relevant information about historical buildings and the past can be found only on display boards or archive exhibition halls, with limited display modes. Obtaining information through QR code breaks through the shackles of traditional media. By scanning a QR code linked to a created historical building display page, a voice introduction and dynamic display can be accessed.

The public participation platform for "Slow Products in Shanghai Hunan Block" is managed and operated by the Hunan Street Office. The aim is to build a public platform for intelligent communities. The platform and consists of three main sections: The first is "Historical Hunan", which mainly records historical buildings and information for the convenience of the public. The second is "enjoy Hunan happily", which integrates data on activities and special services of community and municipal cultural centres, and characteristic businesses to facilitate residents' lives and cultural leisure activities. The third is "Impression of Hunan", which is an interactive platform for residents and tourists to share their memories of the community by photos, travel notes, paintings, videos, and other means, and to make suggestions on the development and renovation of the community so that the public can participate in the development process.

\begin{tabular}{|c|c|c|c|c|}
\hline Platform Type & Platform Name & Participation Mode & Participation Subject & Contents of Public Participation \\
\hline $\begin{array}{c}\text { Historical Resource } \\
\text { Management }\end{array}$ & $\begin{array}{l}\text { Beijing Historical and } \\
\text { Cultural Heritage APP }\end{array}$ & APP Application & Social Public & $\begin{array}{l}\text { 1. Interview and Understanding of Cultural Heritage Information } \\
\text { 2. Supervision and Protection of Cultural and Recreational Sites }\end{array}$ \\
\hline $\begin{array}{l}\text { Planning Design and } \\
\text { Implementation } \\
\text { Management }\end{array}$ & $\begin{array}{c}\text { Community Planning Participation } \\
\text { Discussion Website of Beijing Bell } \\
\text { and DrumTower Renovation } \\
\text { Project WEBGIS }\end{array}$ & WEBGIS Webpage & $\begin{array}{l}\text { Non-Governmental Organizations } \\
\text { Experts and Scholars } \\
\text { Student Teams } \\
\text { Residents }\end{array}$ & $\begin{array}{l}\text { 1. Non-governmental organizations such as social teams established } \\
\text { historical block information database through social research } \\
\text { 2. The public through the network platform to understand the condition } \\
\text { of block renovation and preservation of historical resources, } \\
\text { and to discuss hot issues }\end{array}$ \\
\hline $\begin{array}{l}\text { Cultural Activities } \\
\text { and Tourism } \\
\text { Development }\end{array}$ & $\begin{array}{l}\text { Slow Products in Shanghai } \\
\text { Hunan Block }\end{array}$ & $\begin{array}{l}\text { WeChat Public } \\
\text { Number }\end{array}$ & $\begin{array}{l}\text { Residents } \\
\text { Tourists } \\
\text { Government } \\
\text { Art Teams }\end{array}$ & $\begin{array}{l}\text { 1. Obtain historical building information through two-dimensional } \\
\text { codes } \\
\text { 2. Participate in community life and cultural activities } \\
\text { 3. Save and upload community memories and make suggestions }\end{array}$ \\
\hline
\end{tabular}

Table 1 Case Comparison of Public Participation Platform in Data Age (Source: Author)

\section{Conclusion}

Historical block protection includes the material cultural heritage of the block and the social structure of the block. It is a complicated social management process, and it is necessary to invite multiple subjects to participate in the process of the management and renewal of historical blocks. At the same time, according to the 'ladder theory' of citizen participation (Arnstein, 1969), public participation in historic block protection requires that the public participates in the process of collecting historical information and planning and design related to historical blocks. As the conditions for public participation in China are not yet ripe, it is necessary to guide and standardize the content and forms of public participation through building a public participation platform.

In the data age, public participation platforms for historical block protection use new media and technologies such as WeChat, microblogs, and APP. There are three stages of public participation: the collection and display of historical information, planning design and 
implementation management, and discussion of the block culture and tourism development. Establishment of such a platform will enable the public to shift from passive to active participation and from lagged participation to synchronized participation. Based on cloud technologies such as cloud computing and cloud storage, as well as "micro" technologies such as lightweight platforms, low-cost equipment, and low learning costs, users can simply and quickly participate in historical block protection anytime, anywhere.

With the development of society and the strengthening of consciousness of public participation, in the process of historical protection and cultural inheritance of the city, individuals and teams play a considerable supporting role and have made significant contributions to the protection of urban historical culture. How to integrate the resources and strengths of the government, related institutions, and the public to achieve a "crowdfunding" protection participation model is the focus of the construction of a public participation platform for historical block protection in the data age. This requires the government's "top-down" guidance and the "bottom-up" resource construction of the public from all walks of life. The construction process of the platform itself is also the content of public participation. Only by allowing the public to participate in every process of the protection of historical blocks and platform construction can social resources be effectively integrated to achieve substantive participation of the public.

\section{Reference}

Wei, Jia; Wang, Peng; Wang,Wei (2015)" The Mechanism and Countermeasures of New Media Involvement in Public Participation in Historical Block Protection", Annual National Planning Conference, China, Urban Planning Society of China.

Cheng, Hui; Mao, Mingrui; Yu, Wencheng (2015) “Internet public participation in urban rural planning", Planners, Vol. 31 No. 11 (November).

Liu, Jing (2007) "The origin of public participation and its development in historical and cultural heritage protection", Sichuan Architecture, Vol. 27 No. 1 (February).

Feng, Jiaqi; Li, Jingsheng (2013) “The public participation in the urban historical block's renaissance: Beijing case”, Planners, Vol. 29 No. S2 (July).

Dai, Jia; Zeng, Fanxu; Huang, Shuo (2014) “Rumor propagation in the shadow of the environment: the enlightenment of PX event", Journal of China University of Geosciences(Social Sciences Edition), Vol. 14 No. 1 (January).

Zhou, Kai; Yan, Yan; Song, Bin (2012) "A web-based information exchanging and public participation framework under informationism", Urban Planning International, Vol. 27 No.2(April).

Brown, G; Raymond, CM (2013) "Methods for identifying land use conflict potential using participatory mapping", Landscape \& Urban Planning, Vol. 122 No.122(November).

Arnstein, S R; (1969) "A ladder of citizen participation", Journal of the American Institute of planners, Vol. 35 No.4(July). 
Lane, M B; (2005) "Public participation in planning: an intellectual history", Australian Geographer, Vol. 36 No.3(November).

Choguill, M B G; (1996) "A ladder of community participation for underdeveloped countries", Habitat international, Vol. 20 No.3(September).

Chu, Yan;Mao, Mingrui; Tian, Le; Wang, Chaolun (2015) "CitylF planning cloud platform", Landscape Architecture Frontiers, Vol.3 No.3(May).

Hu, Jiapei; Hu Shuyang; Gao Zhihui (2014) "From Symbol to Essence: Public Participation in the Reconstruction of Historical Streets--Taking Beijing Juer Hutong and Yangzhou Cultural Street as Examples", Annual National Planning Conference, China, Urban Planning Society of China.

Wu, Zuquan (2014) "Effect of third-party participation in urban planning: a case study on enning road in Guangzhou", City Planning Review, Vol.38 No.2(February).

Macaulay, A C; Commanda, L E; Freeman, W L; et al (1999) “Participatory research maximises community and lay involvement", Bmj, Vol.319 No.7212(September).

Xu, Feng; Liu,Tao (2012) "Public participation in planning in Canada and its enlightenment to china”, Urban Planning International, Vol.27 No.1(February).

Liang, Jing (2014) "Study on the renaissance of historic districts in Taiwan from the perspective of community construction--Taking the historic district of the Dadaocheng in Taipei as an Example", Annual National Planning Conference, China, Urban Planning Society of China.

Yu, Tao (2014) "The case analysis of ngos participating in Beijing old city protection", Beijing Planning Review, Vol.28 No.5(September).

$\mathrm{Yu}$, Wencheng; Mao, Mingrui (2015) "The large number is the opportunity and response of the time planning public participation", Urban and Rural Development, Vol.60No.11(November). 